\title{
High Sensitivity Resonance Frequency Measurements of Individual Micro-Cantilevers Using Fiber Optical Interferometry
}

\author{
Thomas Duden ${ }^{*}$ and Velimir Radmilovic ${ }^{*}$ \\ ${ }^{1}$ National Center for Electron Microscopy, Lawrence Berkeley National Laboratory
}

We describe a setup for the resonance frequency measurement of individual microcantilevers. The setup displays both high spatial selectivity and sensitivity to specimen vibrations by utilizing a tapered uncoated fiber tip. The high sensitivity to specimen vibrations is achieved by the combination of optical Fabry-Perot interferometry and narrow band RF detection. Wave fronts reflected on the specimen and on the fiber tip end face interfere, thus no reference plane on the specimen is needed, as demonstrated with the example of freestanding silicon nitride micro-cantilevers. The resulting system is integrated in a DB-235 dual beam FIB system, thereby allowing the measurement of micro-cantilever responses during observation in SEM mode. The FIB was used to modify the optical fiber tip. At this point of our RF system development, the microcantilevers used to characterize the detector were not modified in situ.

PACS 07.60.Ly, 42.81.Pa, 62.25.-g

\footnotetext{
*Coresponding authors: TDuden@lbl.gov (T. Duden) or VRRadmilovic@lbl.gov (V. Radmilovic)
} 


\section{Introduction}

In current integrated sensor devices, MEMS and NEMS play an increasingly important

role. They allow the design of miniaturized balances ${ }^{12}$, chemical and biological sensors ${ }^{3}$ and a variety of other applications.

For the development of a specific device, its mechanical and electrical properties can be assessed by measuring its resonance frequency, damping and stress related frequency shift.

The systematic study of e.g. cantilever aspect ratios and Q factors (the ratio of energy stored to energy lost per cycle) is practically done by manufacturing a whole array of devices with changing dimensions on a single substrate, and to test their characteristic properties during one instrumental session.

For such a measurement, the excitation can be delivered by a piezoelectric actuator, onto which the monolithic specimen is attached. Because all the structures are then moved at the same time, the selection of an individual device has to be achieved by the detector. It should also be highly sensitive for the resulting small vibration amplitudes, and it is desirable to have a high bandwidth allowing for a large variety of materials and dimensions.

Furthermore, because it is easy to excite the structures in modes other than the fundamental, the envelopes of the resulting oscillations should be monitored by an independent imaging method. 


\section{Experimental}

To realize all of the outlined requirements in one setup, we decided to integrate a detection system into the NCEM dual beam FIB. This enables imaging in SEM and ion beam mode and adds FIB machining capabilities for device tuning and optical fiber modification.

To achieve a high bandwidth, an optical pickup was utilized, using near-IR LASER radiation. We also selected a fiber based setup which eliminates external optical adjustments. Only the detector tip needs to be brought to the specific micro-cantilever by a micromanipulator.

To obtain the necessary spatial selectivity, the end of the fiber detector was pulled into a taper. This method is commonly used in scanning near-field optical microcopy, in order to achieve a lateral resolution in the sub wavelength regime. Usually, a metal coating with a small aperture is brought onto the taper tip, allowing only evanescent waves to emerge from the end face, therefore circumventing the diffraction limit ${ }^{4}$. In our setup, because the backscattered light from the micro-cantilever interferes with the wave front reflected on the tip end face, sub wavelength micro-cantilever vibrations can be detected.

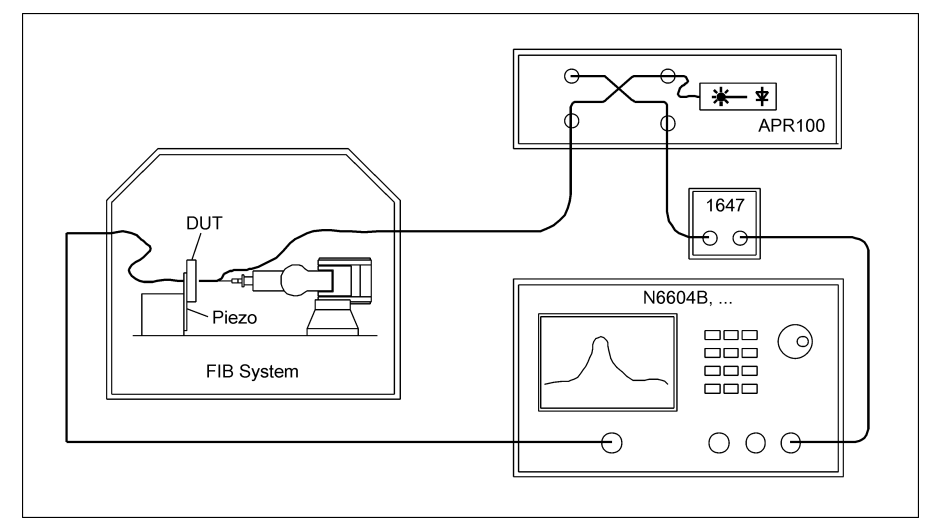

Figure 1: Schematic of experimental setup. 
The optical system incorporates an Attocube ARP-100 laser detector coupler. From the unit, the 2:2 fiber coupler and the infrared diode laser (wavelength $1310 \mathrm{~nm}$, power per output port $0.5 \mathrm{~mW}$ ) were used. As a detector we selected the New Focus Photodiode Detector model 1647. All components are plugged together using FC fiber connectors and vacuum feedthroughs. The signal from the photo detector is fed into a universal spectrum and network analyzer, model Agilent $\mathrm{N} 9320 \mathrm{~A}^{\circledR}$. The optical fiber probe was

formed using $\mathrm{CO}_{2}$ laser heating, with the P-200 Sutter Instruments fiber puller ${ }^{\circledR}$. To avoid charging, the optical fiber tip was covered with aluminum film using PVD (physical vapor deposition) system. It was subsequently cut in the FIB perpendicular to the fiber axis, to achieve a clean and optically transparent end face. Any contamination formed on the optical fiber end face was removed in-situ using focused ion beam. The tapered fiber was attached to a Kleindiek Micromanipulator MM3A-EM ${ }^{\circledR}$ for positioning.

\section{Results and discussion}

The described system (schematically shown in Figure 1) has been set up and used at the NCEM dual beam FIB. The specimen and the tapered optical fiber, which were used to characterize the RF setup are shown in Figure 2. The chip mounted silicon nitride cantilevers had resonant frequencies ranging from 1 to $100 \mathrm{kHz}$. The thickness of the cantilevers was in the range from 530 to $580 \mathrm{~nm}$, the width about 2 microns. The lengths ranged from 100 to 400 microns. They were excited mechanically by means of piezo discs attached to a FIB sample holder. The sample holder and the sample stage were not noticeably vibrating when the micro-cantilevers were excited, which was evident from 
the direct SEM image. The $\mathrm{S}_{3} \mathrm{~N}_{4}$ micro-cantilevers are fabricated by the micromachining using optical lithography ${ }^{56}$. For the first measurements a monofrequent excitiation source was selected, using a Stanford Research DS345 signal generator, which allows precise excitation amplitude and frequency control.

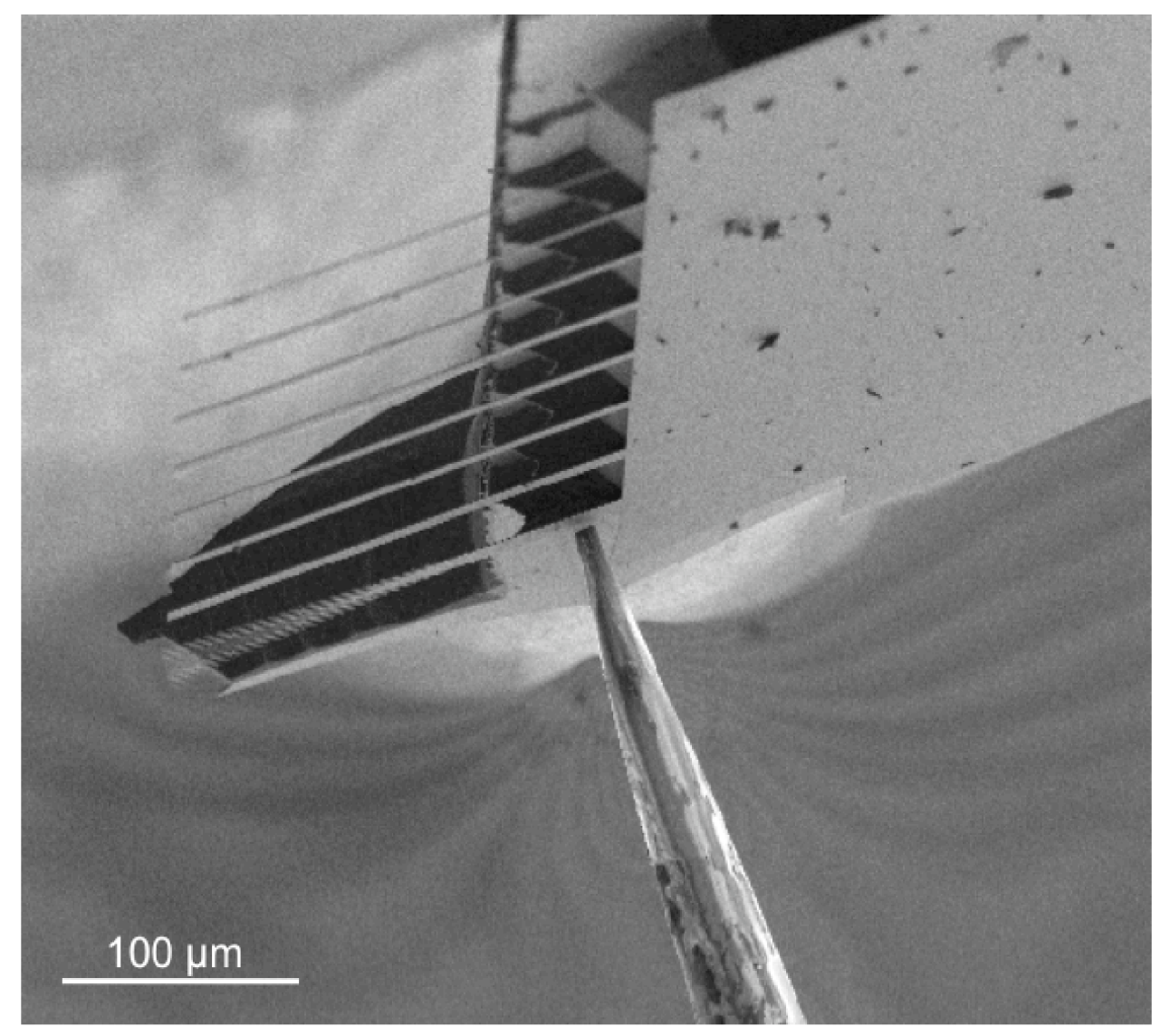

Figure 2: View of the tapered optical fiber positioned to collect the signal from a resonating micro-cantilever.

Figure 3 shows the detector signal from the arrangement shown in Figure 2 with the described excitation source. It is clear that this configuration provides a signal level of several order of magnitude above noise floor. 


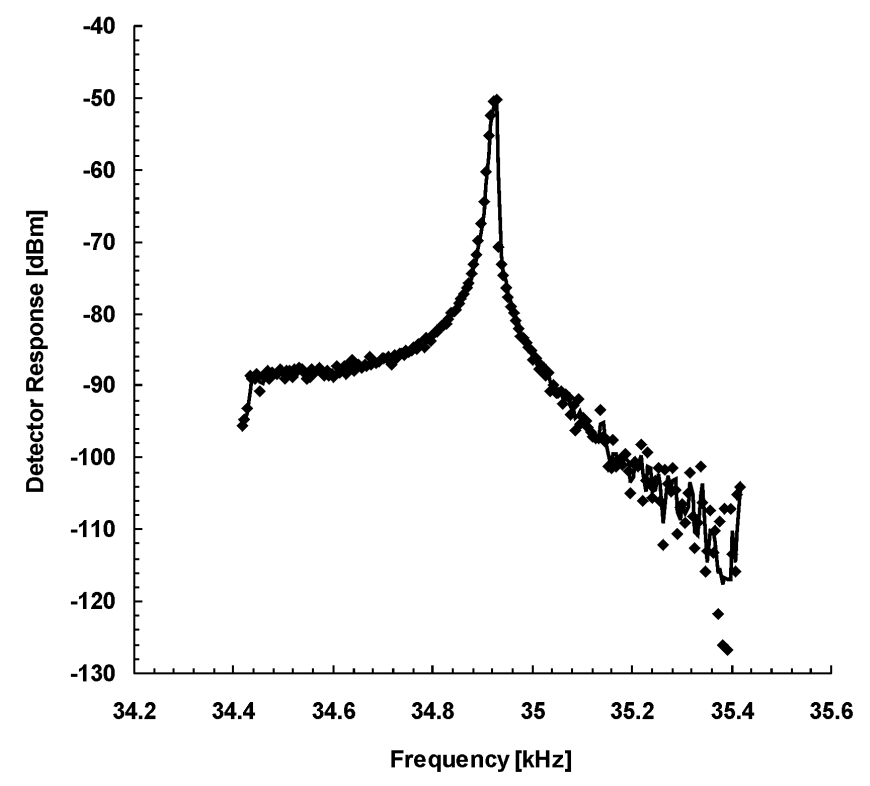

Figure 3: Signal from the arrangement in Fig. 2. For this micro-cantilever, the excitation source was a signal generator with a fixed frequency. The fiber had an end face diameter of $\sim 0.8 \mu \mathrm{m}$.

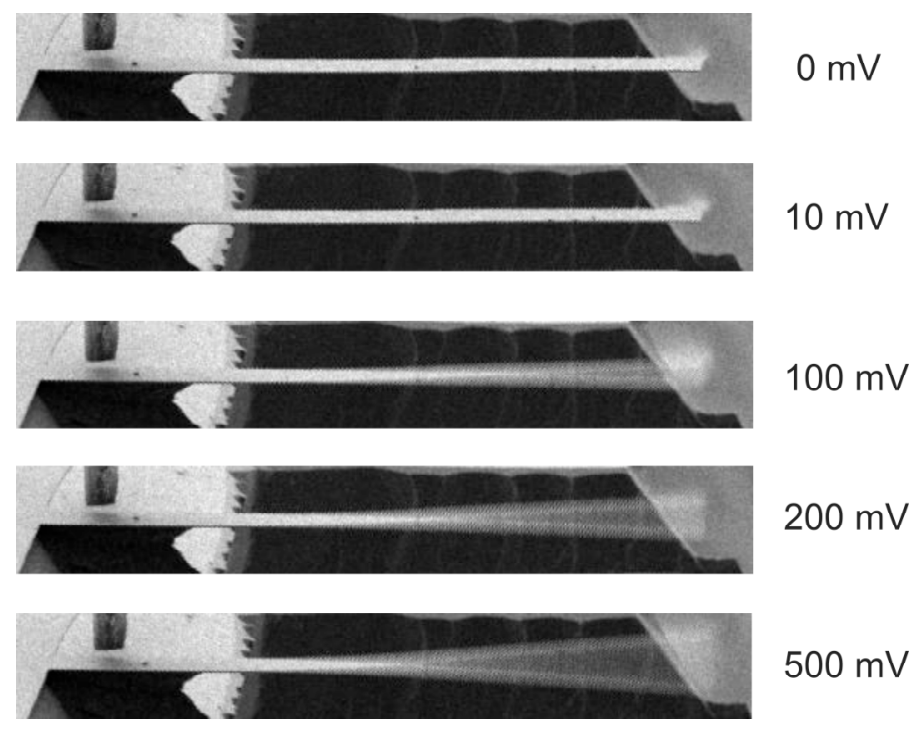

Figure 4: Micro-cantilever at different excitation amplitudes. Above the support lamella on the left hand side, the optical fiber is in close proximity to the surface 
The first measurement discussed here targeted a part of the micro-cantilever supported by a silicon lamella and displayed no resonantly amplified vibrations during the measurement (Figure 4). Thus, only the vibrations stemming from the piezo support are measured. The response curve is shown in Figure 5. The response signal amplitude calculated from the spectra scales surprisingly linear with the excitation amplitude. Note that in the SEM images, no vibration of the structure is yet detectable. The resolution of the SEM images was set to $0.45 \mu \mathrm{m} /$ pixel. Clearly, the probe is sensitive to vibrations well below the wavelength of the irradiating light. To evaluate the lower sensitivity limit, the displacement of the piezo actuator was measured. At higher excitation voltages, this is possible by using the SEM, directly imaging the envelope of the actuator. The found values were linearly extrapolated to zero, to account for displacements that were below the detection limit of the SEM. Using the resulting extrapolation coeffient, at a frequency of $30 \mathrm{kHz}$, a vibrational amplitude of about $1.26 \mathrm{~nm}$ delivered a signal of $-88.9 \mathrm{dBm}$ at the detector. The noise floor was $-103 \mathrm{dBm}$, e.g. the signal-to background ratio was about 10 for our smallest excitation voltage. This enables the careful statement that vibration amplitudes below $1 \mathrm{~nm}$ should still be above the detection limit of this method. The upper limit of the dynamic range is encountered by contact between the cantilever and the fiber tip, which usually caused one or, at higher amplitudes, several dips in the spectrum, located at or around the center frequency. The amplitude of the object should thus not reach or exceed the tip-object distance, which was about 3 microns in our case. It is worth mentioning that the scanning beam can influence the spectrum by charging, which can be avoided by beam blanking after the tip is positioned. 


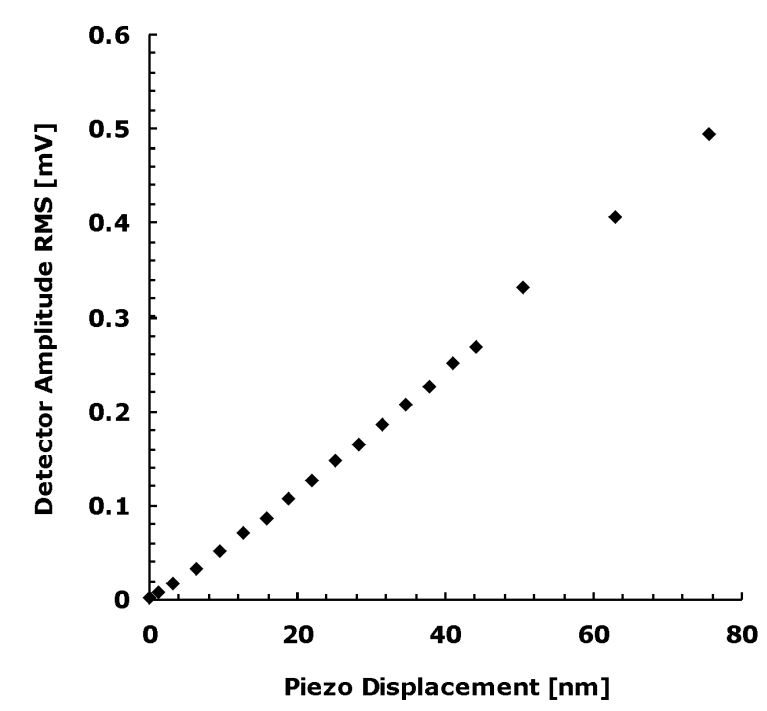

Figure 5: Detector response on different excitation amplitudes for the arrangement as shown in Fig. 4 .

In a second run, the vibration amplitude was measured along the cantilever as a function of distance from a cantilever undercut. The resulting profile is shown in Figure 6. The comparison with the images given in Figure 3 shows that the vibration amplitude in the region below 15 um (which corresponds to the support by the lamella) is constant and increases as the free part of the beam is reached. At higher excitations, the relative increase of the measured oscillation amplitude gets smaller because the cantilevers respond nonlinear and their resonance shifts upwards, away from the excitation frequency. 


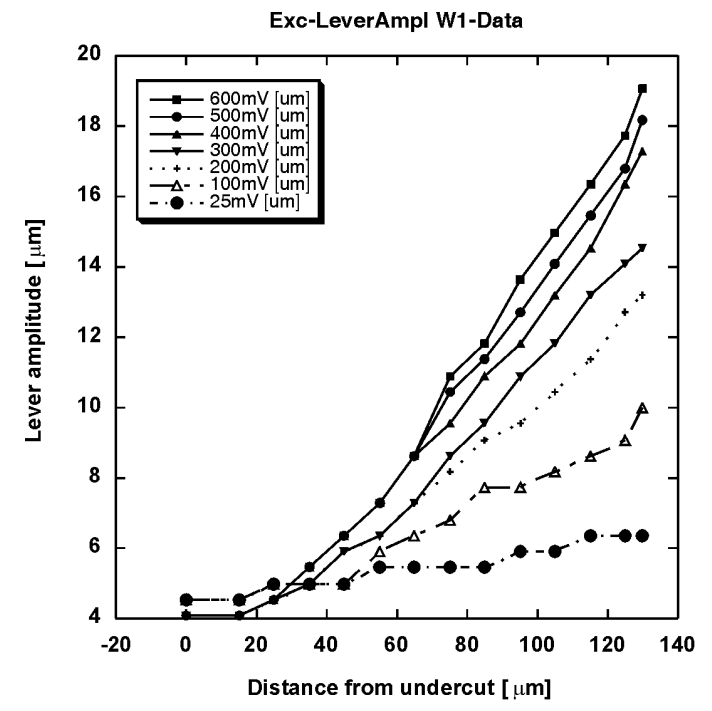

Figure 6: Measured detector output along the cantilever from Fig. 4. Between 0 and $15 \mu \mathrm{m}$, the cantilever is supported by the lamella below the cantilever. Due to nonlinear cantilever response, the gain in amplitude decreases at higher excitations. In the third run, the lateral position of the probe was moved across the sample to determine the lateral resolution of the probe. Figure 7 shows measured profile across cantilever shown in Figure 3. It is to be noted that for

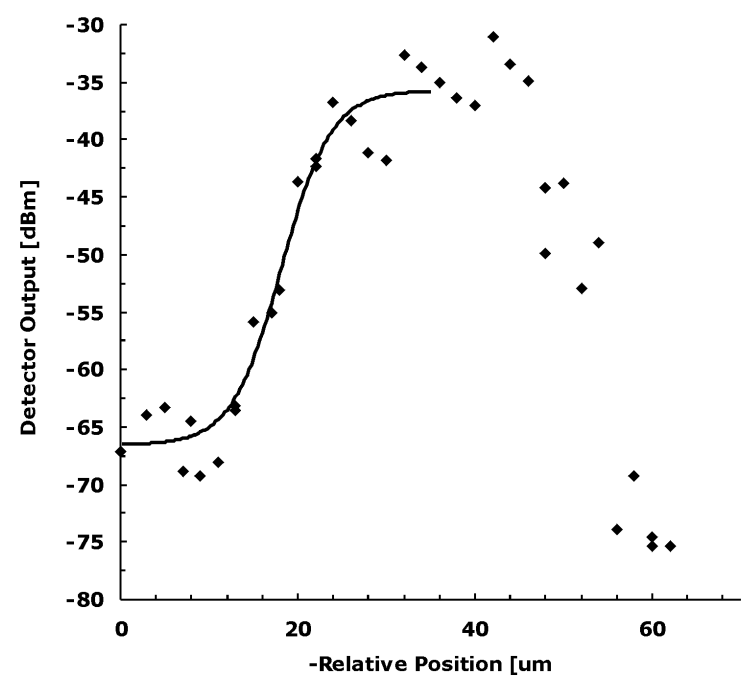

Figure 7: Measured profile across the cantilever from Fig. 3. The probe collects light at ca. 3.5 um above the cantilever surface. The line is a fitted hyperbolic tangent function. 
specimen of the size similar to the wavelength of the light used, diffraction modifies the amplitude of the backscattered intensity. From the fitted hyperbolic tangent function, a lateral resolution of $7.7 \mu \mathrm{m}$ was extracted $(20 \%-80 \%)$.

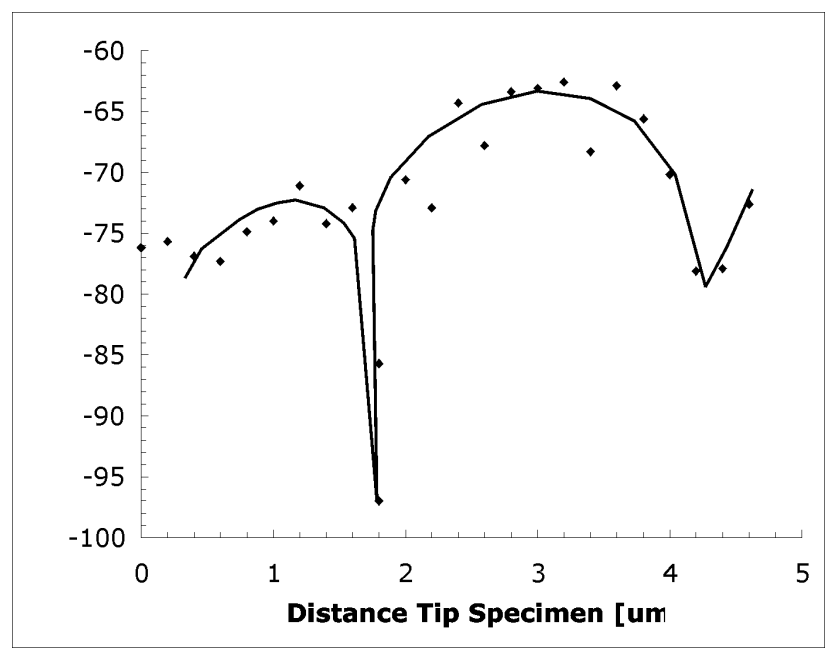

\section{Figure 8: Detector response on variation of tip-specimen distance (the line is to} guide the eye).

To characterize the measured signal sensitivity in dependence of the specimen distance, we varied the tip-specimen distance at constant excitation. The resulting curve is displayed in Figure 8. The curve shows a clear and reproducible dip at about $1.8 \mu \mathrm{m}$ and had an overall maximum at $3.4 \mu \mathrm{m}$ distance. Note that the distance was recalculated from the images, taking a sample tilt into account that was determined later on. The optimum can result from the overlay of several influences: (a) the diffraction modifying the backscattered wave intensity distribution, (b) the balance of the light wave amplitudes backscattered from the specimen and from the probe tip end face (resulting in different fringe visibilities), and probably (c) parasitic interference stemming from the various $\mathrm{FC}$ connections in the fiber path. The dip could be the consequence of measuring at a fringe maximum, where vibrations at a frequency $\mathrm{f}$ would result in intensity variations of $2 \mathrm{f}$. 
We have presented a useful approach for a versatile vibration measurement setup. It is rugged and needs, besides the tip positioning, no other adjustments. The high spatial selectivity of the device enabled the characterization of individual micro-cantilevers and a very sensitive evaluation of the present vibrations. Through the combination of optical interferometry and narrow band RF detection, we were able to demonstrate an exceptional combination of spatial and vibrational sensitivity.

Acknowledgments: The authors acknowledge support by the US Department of Energy under Contract \#DE-AC02-05CH11231. The authors want thank to Dr. Bojan Ilic of Cornell NanoScale Science and Technology Facility at Cornell University for providing cantilever samples, professor Kahled Karrai and Dr. Dirk Haft of Attocube, Dr. David Hwang of UC Berkeley and Colin Ophus and Erik Luber of University of Alberta for helpful discussions. The authors also like to thank Dr. Jack Belgum of Sutter Instrument for providing their fiber puller to create high quality optical fiber probes.

\section{References}

${ }^{1}$ Y. T. Yang, C. Callegari, X. L. Feng, K. L. Ekinci, and M. L. Roukes, Nano Letters 6, $583(2006)$.

2 Takahito Ono, Xinxin Li, Hidetoshi Miyshita, and Mayasoshi Esashi, Review of Scientific Instruments 74, 1240 (2003). 
${ }^{3}$ Nickolay V. Lavrik, Michael J. Sepaniak, and Panos G. Datskos, Review of Scientific Instruments 75, 2229 (2004).

${ }^{4}$ E. L. Buckland, P. J. Moyer, and M. A. Paesler, J. Appl. Phys. 73, 1018 (1993).

${ }^{5}$ B. Ilic, D. Czaplewski, H. G. Craighead, P. Neuzil, C. Campagnolo, and C. Batt, Appl. Phys. Lett. 77, 450 (2000).

${ }^{6}$ L. Sekaric, D.W. Carr, S. Envoy, J.M. Parpia, and H.G. Craighead, Sens. Actuators, A 101, 215 (2002). 